\title{
Geometric Constraints and Motion Branch Variations for Reconfiguration of Single-Loop Linkages with Mobility One
}

\author{
Ketao Zhang ${ }^{\mathrm{a}, *}$, Jian S. Dai ${ }^{\mathrm{a}}$ \\ ${ }^{a}$ Centre for Robotics Research, Kings College London, University of London \\ Strand, London WC2R 2LS, UK
}

\begin{abstract}
This paper explores essence of geometric constraints induced reconfiguration of single-loop kinematic chains with mobility one. Reconfigurable kinematic chains are firstly classified into four categories in light of variations of parameters in the Chebychev-Grübler-Kutzbach mobility criterion. Within these four categories, single-loop kinematic chains with mobility one but distinct motion branches are further classified in accordance with degeneration of degree-of-freedom of certain revolute joints. With the essence of reconfiguration, the interrelationship of motion-branch changes through constraint singularity induced transitory positions of reconfigurable single-loop linkages is revealed in the context of reciprocity of screws. Four basic geometric constraints leading to transitory positions are explored by analysing the Bennett plano-spherical linkage, a kinematic embodiment of Grassmann varieties. Geometric constraints induced screw-system variation and motion branch changes of a novel asymmetric 7R linkage and the line- and plane-symmetric Bricard $6 \mathrm{R}$ linkage with capability of reconfiguring their motion branches are subsequently analysed for interpreting the fundamentals explored.
\end{abstract}

Keywords:

Reconfiguration, mobility criterion, constraint singularity, screw system, overconstrained $6 \mathrm{R}$ linkage, reconfigurable $7 \mathrm{R}$ linkage

\footnotetext{
Final submission.

*Corresponding author

Email addresses: ketao.zhang@gmail.com (Ketao Zhang), jian.dai@kcl.ac.uk (Jian S. Dai)
} 


\section{Introduction}

Reconfigurable mechanism defines a kind of mechanism which possesses capability to change its global mobility and motion characteristics. This capability distinguishes reconfigurable mechanisms from conventional mechanisms with invariant kinematic structure and functionality. With reconfigurability and variable motion characteristics, reconfigurable mechanisms can be developed into multifunctional robot manipulators, medical devices and manufacturing devices to adapt to unexpected conditions and to perform multitasks that would be challenge for conventional mechanisms.

In the last two decades, typical mechanisms with reconfigurability have been investigated by revealing their inherent properties of structure reconfiguration and analyzing the apparent phenomenon of mobility change. Wohlhart [1] presented a type of linkage that is able to permanently change their global mobility by passing a singular position and termed the peculiar property kinematotropy. For a kinematotropic mechanism, the mobility is changing when the mechanism reconfigures to distinct motion branches [2] separated by singular positions [3]. Around the same time, Dai [4] developed reconfigurable assemblies and packaging systems for folding decorative gifts and origami-type boxes, leading to exploration of the metamorphic mechanism [5]. Metamorphic mechanisms are capable of changing their structure and subsequently mobility resorting to either link annex or joint variation at typical configurations. The apparent mobility change of a metamorphic mechanism is accompanied by kinematic structure variation in the form of changes of effective links and kinematic joints $[6,7]$. Along with kinematotropic linkages and metamorphic mechanisms, mechanisms with different motion branches, named as operation modes [8], were revealed and such kind of mechanism with multiple motion branches can undergo a variety of transformations by passing its constraint singularities. The study of this type of mechanisms mainly focuses on the variation of operation modes of its platform, a particular selected output link of the mechanism [9]. Apart from the above three types of reconfigurable mechanisms, mechanisms with variable topologies $[10,11]$ characterized by topological structure changes during operation were explored for dealing with complicated tasks.

Comparing to the broad study of reconfigurable parallel mechanisms and robots [12-19], single-loop mechanisms capable of reconfiguring their kine- 
matic structure had rarely been explored and only a few of typical cases were presented recently. Galletti and Fanghella [20] presented four basic kinematotropic single-loop kinematic chains and addressed changes in pair connectivity of linkages with constant numbers of degree(s) of freedom. In light of the concept of metamorphosis, Zhang, Dai and Fang [21] presented a novel metamorphic 8R linkage inspired by artistic kirigami. Distinct motion branches were derived form the $8 \mathrm{R}$ linkage resorting to both physical and geometric constraints. This study further led to identification of two specific types of line-symmetric overconstrained 6R linkages. Kuo and Yan [22] investigated configuration syntheses and analyses of mechanisms with variable topologies and presented typical single-loop linkages with different stationary configurations. Kong and Huang [23] synthesized and constructed single-loop mechanisms with two operation modes employing basic single degree-of-freedom (DOF) linkages such as planar 4R, spherical 4R, Bennett $4 \mathrm{R}$ linkage and paradoxical $5 \mathrm{R}$ and $6 \mathrm{R}$ linkages. This study further extended to single-loop $7 \mathrm{R}$ mechanisms with multi-operation mode [24, 25]. Zhang, Müller and Dai [26] explored a novel reconfigurable 7R linkage that is able to evolve to both overconstrained $6 \mathrm{R}$ linkage and planar $4 \mathrm{R}$ linkages. Chen and You [27] investigated bifurcations of two-fold symmetrical 6R deployable linkage using singular value decomposition of the Jacobian matrix of closure equations. Chen and Chai [28] put forward the study further on the bifurcated motion of a special line and plane symmetric Bricard linkage [29]. Further to above overconstrained 6R linkages with bifurcated motion branches, Zhang and Dai [30] further explored trifurcation of the Bennett plano-spherical hybrid linkage based on reciprocity of screws [31]. The 6R linkage allows distinct 1 DOF motion branches with geometrically restricted kinematic joints and transition between these motion branches by passing the singular position. A new method based on intersection of surfaces generated by kinematic dyads and the singularities of these surfaces was developed for identifying reconfigurable mechanisms [32].

Though aforementioned work on reconfigurable single-loop kinematic chains have been made, most of the efforts dedicated to reconfiguration analysis of individual mechanism and to designs combining existing mechanisms. Fundamentals for both kinematics analysis and structure synthesis of reconfigurable single-loop mechanisms are yet systematically explored.

Towards the theory of freedom to move in systems of articulated rigid bodies, screw theory [31, 33-35] and closed-form solutions [36, 37] provided a mathematical framework for both kinematics analysis and structure synthesis 
[38-40]. In terms of interrelationship [41, 42] between a screw system and its corresponding reciprocal system, a screw-system approach for rigid-body motion analysis of kinematic chains particularly overconstrained mechanisms was developed [43].

In light of the theory of freedom to move in systems composed of articulated rigid bodies, this paper is to investigate the essence of geometric constraints induced screw-system-variation and reconfiguration of single-loop mechanisms. The study firstly classifies reconfigurable single-loop kinematic chains in terms of variables $d, n, g$ and $f_{i}$ of the general Chebychev-GrüblerKutzbach mobility criterion. Looking closely at variation of parameter $d$, the interrelationship of screw-system variation corresponding to motion-branch change via constraint singularities of the single-loop reconfigurable mechanism is explored. With revealed essence for reconfiguration of single-loop mechanisms, four types of geometric constraints leading to transitory positions are explored by analysing motion branch changes of the Bennett planospherical linkage in conjunction with Grassmann varieties. The presented interrelationship and geometric constraints based approach are further interpreted by analysing a novel asymmetric 7R linkage and the line- and planesymmetric Bricard 6R linkage.

\section{Screw-system and Motion Branch Variations of Reconfigurable Mechanisms}

In this section, underlying principles resulting in structure and mobility changes of reconfigurable single-loop mechanisms are exploited in terms of screw theory and Grassmann varieties [44] of line geometry.

\subsection{Mobility Criteria Based Classification of Reconfigurable Mechanisms}

Mobility of a mechanism is generally calculated in terms of the ChebychevGrübler-Kutzbach criterion $[45,46]$ expressed in the formula

$$
m=d(n-1) \sum_{i=1}^{g}\left(d-f_{i}\right)=d(n-g-1)+\sum_{i=1}^{g} f_{i}
$$

in which $m$ is the mobility of a mechanism, $d$ is the degrees-of-freedom of the space in which the mechanism works, $n$ is the number of bodies connected by $g$ joints and $f_{i}$ is the connectivity or degree(s)-of-freedom of the $i$ th joint. 
The mobility formula in Eq. (1) counts the numbers of bodies (links), joints and the degrees-of-freedom of joints. It determines mobility of a kinematic chain in general but may break down for overconstrained mechanisms and predict less mobility due to peculiar geometric conditions and dimensions of these mechanisms.

Taken parameter $d[47,48]$ in Eq. (1) as the order of mechanism motionscrew system, modified mobility criterion [43] was then proposed by extending $d$ to other values less than 6 and by accounting for redundant constraints for mobility prediction of mechanisms with peculiar geometric conditions.

In contrast to conventional mechanisms performing a sole function, a reconfigurable mechanism is able to perform multiple functions by passing transitory positions and switching between distinct motion branches. The mobility $m$ and motion characteristics of a reconfigurable mechanism are thus changing in accordance with the motion branch changes. According to the mobility criterion, the global mobility of each motion branch and the instantaneous mobility at a transitory position are defined by parameters $n$, $g, f_{i}$ and $d$. Changes to any of these variables lead to variation of the global mobility and motion characteristics of the mechanism.

Table 1: Classification of reconfiguration in terms of parameters in mobility criterion

\begin{tabular}{|c|c|c|c|c|c|c|c|}
\hline Category & Parameters & $\begin{array}{l}\text { Physical } \\
\text { meaning }\end{array}$ & $\begin{array}{l}\text { Form of } \\
\text { changes }\end{array}$ & \multicolumn{3}{|c|}{ Type of Reconfiguration } & Essence \\
\hline 1 & $n$ & $\begin{array}{l}\text { Number of } \\
\text { bodies }\end{array}$ & $\begin{array}{l}\text { Link annexing/ } \\
\text { releasing }\end{array}$ & \multicolumn{3}{|c|}{ Metamorphosis } & \multirow{4}{*}{$\begin{array}{l}\text { Physical constraints } \\
\text { at certain configurations }\end{array}$} \\
\hline 2 & $g$ & $\begin{array}{l}\text { Number of } \\
\text { joints }\end{array}$ & $\begin{array}{l}\text { Restricted/ } \\
\text { unrestricted joint }\end{array}$ & Metamor & hosis & & \\
\hline \multirow{2}{*}{3} & \multirow{2}{*}{$f_{i}$} & \multirow{2}{*}{$\begin{array}{l}\text { Degree(s) of } \\
\text { freedom of a } \\
\text { joint }\end{array}$} & Reconfigurable joints & \multicolumn{3}{|c|}{ Metamorphosis } & \\
\hline & & & $\begin{array}{l}\text { Variable kinematic } \\
\text { joints }\end{array}$ & \multicolumn{3}{|c|}{ Variable topologies } & \\
\hline \multirow{4}{*}{4} & \multirow{4}{*}{$d$} & \multirow{4}{*}{$\begin{array}{l}\text { Order of } \\
\text { mechanism } \\
\text { motion-screw } \\
\text { system }\end{array}$} & \multirow{4}{*}{$\begin{array}{l}\text { Mechanism screw- } \\
\text { system variation } \\
\text { and motion branch } \\
\text { change }\end{array}$} & \multirow[t]{2}{*}{$\begin{array}{l}\text { Variant } \\
\text { mobility }\end{array}$} & $\begin{array}{l}\text { Degenerated degree }(\mathrm{s}) \\
\text { of freedom of } \\
\text { certain joints }\end{array}$ & Metamorphosis & \multirow{4}{*}{$\begin{array}{l}\text { Geometric constraints of } \\
\text { kinematic joints }\end{array}$} \\
\hline & & & & & $\begin{array}{l}\text { Original degree(s) } \\
\text { of freedom of all joints }\end{array}$ & $\begin{array}{l}\text { Kinematotropy } \\
\text { Multifurcation } \\
\text { Multi-mode }\end{array}$ & \\
\hline & & & & \multirow[t]{2}{*}{$\begin{array}{l}\text { Invariant } \\
\text { mobility }\end{array}$} & $\begin{array}{l}\text { Degenerated degree(s) } \\
\text { of freedom of } \\
\text { certain joints }\end{array}$ & Metamorphosis & \\
\hline & & & & & $\begin{array}{l}\text { Original degree }(\mathrm{s}) \\
\text { of freedom of all joints }\end{array}$ & $\begin{array}{l}\text { Multifurcation } \\
\text { Multi-mode }\end{array}$ & \\
\hline
\end{tabular}

According to parameters $n, g, f_{i}$ and $d$, reconfiguration of the closed-loop mechanism are classified into four categories in Table 1 . Corresponding to each parameter in Table 1, form of changes and types of reconfiguration are enumerated respectively.

Types of reconfiguration include kinematotropy [1], metamorphosis [5], 
variable topology [10], multifurcation [49] and multi-mode [8, 9]. The metamorphosis, a particular feature in the sense of evolution, characterizes metamorphic mechanisms capable of changing their structure, topological configuration and subsequently mobility. In analogy to metamorphosis, kinematotropy defines the kinematotropic mechanism which permanently changes its global mobility by passing a transitory position. The mechanism with variable topologies are characterized by topological features of variable kinematic joints employed in this kind of mechanism.

Subjects of the present paper are reconfigurable single-loop mechanisms with mobility one in category 4 , of which the reconfiguration is induced by special geometrical arrangement of kinematic joints.

\subsection{Screw-System-Variation in Conjunction with Constraint Singularity of Reconfigurable Mechanisms}

In terms of the screw-system approach [43], mechanism motion-screw system $\mathbb{S}$ and mechanism constraint-screw system $\mathbb{S}^{c}$ form a dual pair and the reciprocity of these two screw systems is defined by

$$
\mathbb{S}^{\mathrm{c}}=\left\{\mathbf{S}^{\mathrm{c}} \mid \mathbf{S}^{\mathrm{c}} \circ \mathbf{S}=0, \forall \mathbf{S} \in \mathbb{S}\right\}
$$

in which $\mathbf{S}^{c}$ and $\mathbf{S}$ represent constraint screws and motion screws, respectively.

For spatial mechanisms, given an motion-screw system of order $d$, the constraint screws reciprocal to the $n$-system form a $(6-d)$-system, meaning

$$
\operatorname{dim}(\mathbb{S})+\operatorname{dim}\left(\mathbb{S}^{\mathrm{c}}\right)=d+\lambda=6
$$

where $\operatorname{dim}(*)$ represents order of a screw system, $d$ and $\lambda$ denote order of mechanism motion-screw system and order of mechanism constraint-screw system, respectively.

When a single-loop mechanism reaches its constraint singularities, the order of mechanism constraint-screw system $(\lambda)$ increases while the order of mechanism motion-screw system $(d)$ decreases reciprocally at these specific configurations. According to the mobility criterion, the decrease of $d$ generally leads to increase of infinitesimal mobility at singular configurations. With consideration of the geometric conditions of kinematic joints of a single-loop mechanism, variation of $d$ and $\lambda$ and resulted reconfigurations by passing a singularity incurred transitory position are summarized in Fig. 1, assuming there are two distinct motion branches apart from the original configuration without losing generality. 


\subsection{Interrelationship of Various Motion Branches: Common Constraint Screw Bases at the Transitory Position}

As illustrated in Fig. 1, screw-system variations result in reconfiguration of a mechanism by passing the transitory position where constraint singularity occurs. In the original configuration, all bodies (links) and kinematic joints of the mechanism are effective. At a transitory position, the order of mechanism constraint-screw system $\mathbb{S}^{c}$ increases by $\tau(\tau>0)$ and becomes $\lambda_{t}=(\lambda+\tau)$. Reciprocally, the order of mechanism motion-screw system $\mathbb{S}$ decreases and becomes $d_{t}=(6-\lambda-\tau)$. Since the mechanism is able to move to at least one more distinct motion branch by passing a transitory position, the constraint-screw bases corresponding to the transitory position span a common constraint-screw system $\mathbb{S}_{t}^{c}$ of all motion branches correlating to the transitory position. In other words, $\mathbb{S}_{t}^{c}, \mathbb{S}_{t i}^{c}$, and $\mathbb{S}_{t j}^{c}$ share same screw bases, assuming $\mathbb{S}_{t i}^{c}$ and $\mathbb{S}_{t j}^{c}$ are constraint-screw systems corresponding to evolved motion branches $\mathrm{MB}_{i}$ and $\mathrm{MB}_{j}$. These common constraint-screw bases of the transitory configuration are reciprocal to mechanism motion-screw systems $\mathbb{S}_{t}, \mathbb{S}_{t i}$ and $\mathbb{S}_{t j}$, where $\mathbb{S}_{t i}\left(\mathbb{S}_{t i} \subset \mathbb{S}_{t}\right)$ and $\mathbb{S}_{t j}\left(\mathbb{S}_{t j} \subset \mathbb{S}_{t}\right)$ are multi-sets of motion screws representing kinematic joints belonging to motion branches $\mathrm{MB}_{i}$ and $\mathrm{MB}_{j}$, respectively.

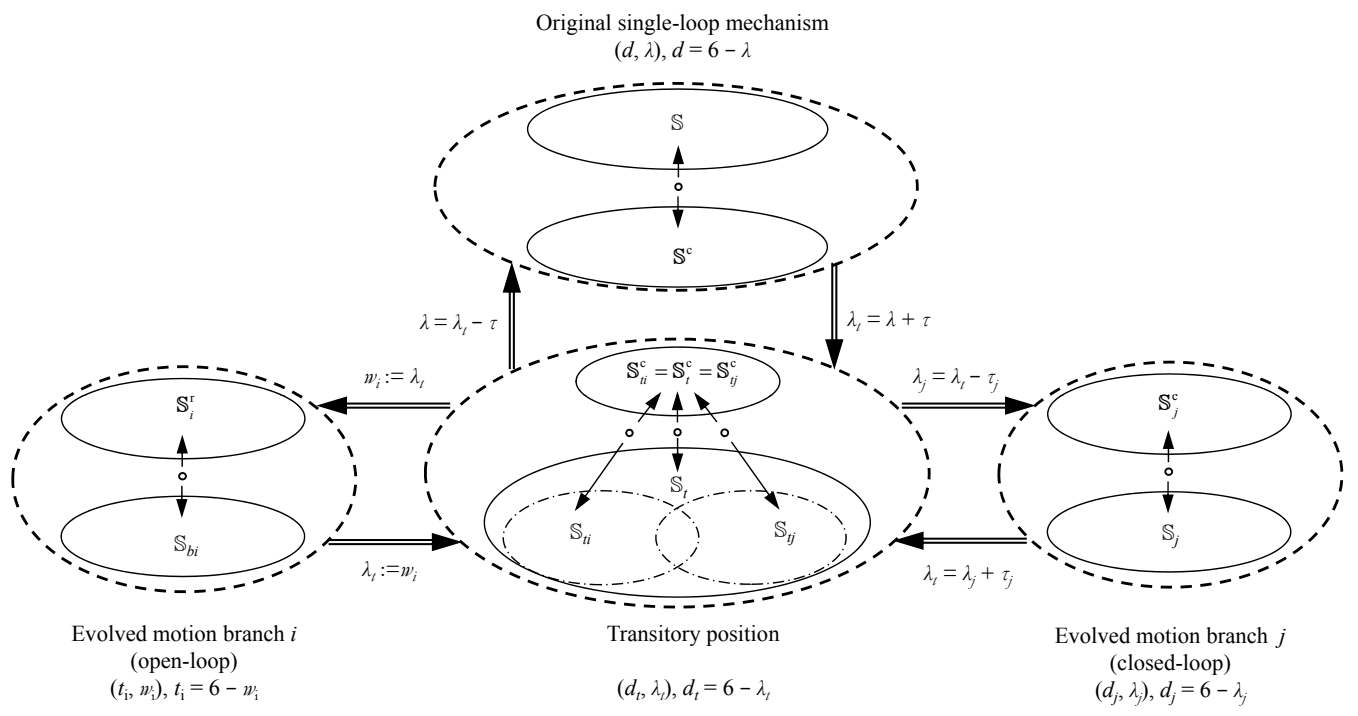

Figure 1: Screw-system-variation via constraint induced transitory position

When the mechanism changes from the transitory position to motion 
branch $\mathrm{MB}_{i}$, which is an evolved serial kinematic chain, the mechanism motion-screw system describing this motion branch is denoted $\mathbb{S}_{b i}$, of which the order $t_{i}$ is determined by the geometry arrangement of active kinematic joints of the serial kinematic chain in a non-singular configuration. Constraint-screw system $\mathbb{S}_{t i}^{r}$ of this motion branch is a span of wrenches reciprocal to motion-screw system $\mathbb{S}_{b i}$. The order of the constraint-screw system is denoted by $w_{i}$ and calculated as $w_{i}=6-t_{i}$.

By passing the transitory position and changing to motion branch $\mathrm{MB}_{j}$ with single-loop topology, the mechanism constraint-screw system of a nonsingular configuration in this evolved motion branch changes to $\mathbb{S}_{j}^{c}$. The order of constraint-screw system decreases by $\tau_{j}\left(\tau_{j} \geq 0\right)$ from that of transitory position and becomes $\lambda_{j}=\left(\lambda_{t}-\tau_{j}\right)$. The mechanism motion-screw system $\mathbb{S}_{j}$ is a span of screws reciprocal to constraint screws and its order becomes $d_{j}=6-\lambda_{j}=6-\lambda_{t}+\tau_{j}$.

Global mobility of evolved serial kinematic chain, $\mathrm{MB}_{i}$, is straight forward by counting effective kinematic joints while the mobility of evolved single-loop mechanism, $\mathrm{MB}_{j}$, is calculated with the mobility criterion in Eq. (1).

\section{Exploration of Grassmann Varieties and Screw System Varia- tions in Motion Branch Reconfigurations}

In this section, the geometric characteristics of three Grassmann varieties [44] which induce motion branch changes are explored by analysing the screwsystem variations of the Bennett plano-spherical hybrid linkage[50] following the principles presented in the above section.

The Bennett plano-spherical hybrid linkage is a typical overconstrained 6R linkage. As illustrated in Fig.2(a), axes of revolute joints $R_{1}, R_{2}$ and $R_{6}$ have common point $A$ and axes of revolute joints $\mathrm{R}_{3}, \mathrm{R}_{4}$ and $\mathrm{R}_{5}$ are parallel. The plane of symmetry determined by axes of joints $R_{1}$ and $R_{4}$ is denoted by $\Pi_{1}$. Axes of joints $R_{2}$ and $R_{3}$ intersect at common point $C$ and axes of joints $R_{5}$ and $R_{6}$ intersect at common point $D$. These two common points are symmetric with respect to plane $\Pi_{1}$. In a general configuration, two coplanar axes of joints $\mathrm{R}_{1}$ and $\mathrm{R}_{4}$ have an instantaneous common point $E$.

A Cartesian coordinate frame $O-X Y Z$ with the origin attached at common point $A$ is set as global frame of the $6 \mathrm{R}$ linkage. The $X$ - and Z-axis are located in symmetric plane $\Pi_{1}$ with axis of $R_{4}$ parallel to $X$-axis and perpendicular to $Z$-axis, $Y$-axis is set following the right-hand rule in Fig.2. 


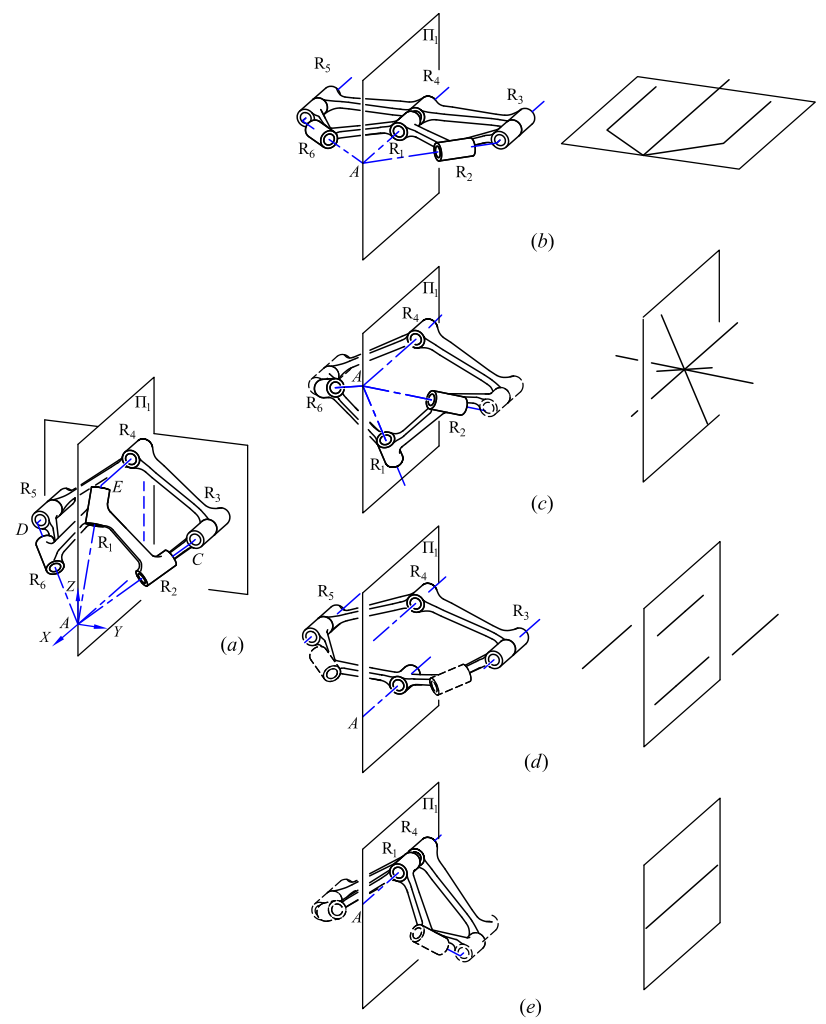

Figure 2: Reconfiguration of the Bennett plano-spherical linkage: (a) a general configuration, (b) coplanar constraint induced singular configuration, (c) $\mathrm{MB}_{1}$, concurrent constraint induced spherical 4R linkage, (d) $\mathrm{MB}_{2}$, parallel constraint induced planar $4 \mathrm{R}$ linkage, (e) $\mathrm{MB}_{3}$, collinear constraint induced serial kinematic chain

With respect to global coordinate frame $O-X Y Z$ in Fig. 2, motion screws of the $6 \mathrm{R}$ linkage in a general configuration span a screw system $\mathbb{S}$, given by

$$
\mathbb{S}=\left\{\begin{array}{l}
\mathbf{S}_{1}=\left[\begin{array}{llllll}
l_{1} & 0 & n_{1} & 0 & 0 & 0
\end{array}\right]^{\mathrm{T}} \\
\mathbf{S}_{2}=\left[\begin{array}{llllll}
l_{2} & m_{2} & n_{2} & 0 & l s \alpha & -l c \alpha
\end{array}\right]^{\mathrm{T}} \\
\mathbf{S}_{3}=\left[\begin{array}{lllllll}
1 & 0 & 0 & 0 & n_{2} & -m_{2}
\end{array}\right]^{\mathrm{T}} \\
\mathbf{S}_{4}=\left[\begin{array}{lllllll}
1 & 0 & 0 & 0 & n_{2}+r c \theta_{40} & 0
\end{array}\right]^{\mathrm{T}} \\
\mathbf{S}_{5}=\left[\begin{array}{lllllll}
1 & 0 & 0 & 0 & n_{2} & m_{2}
\end{array}\right]^{\mathrm{T}} \\
\mathbf{S}_{6}=\left[\begin{array}{llllll}
l_{2} & -m_{2} & n_{2} & 0 & 0 & 0
\end{array}\right]^{\mathrm{T}}
\end{array}\right.
$$


where $r$ is the link length of links between parallel joint axes, $\theta_{40}$ is a variable defined by $\theta_{40}=\left(\theta_{4}-\pi\right) / 2$ and $\theta_{4}$ is the angular displacement of joint $R_{4}$.

The constraint-screw system, a span of constraint screws reciprocal to motion screws in Eq. (4), is derived as

$$
\mathbb{S}^{\mathrm{c}}=\mathbf{S}_{1}^{\mathrm{r}}=\left[\begin{array}{llllll}
1 & 0 & 0 & 0 & 0 & 0
\end{array}\right]^{\mathrm{T}}
$$

Hence, the order of mechanism constraint-screw system of the $6 \mathrm{R}$ linkage in its general configuration in Fig. 2 is one. It further implies that the mechanism motion-screw system in Eq. (4) is a five-system, meaning $\lambda=$ $\operatorname{dim}\left(\mathbb{S}^{c}\right)=1$ and $d=\operatorname{dim}(\mathbb{S})=6-\lambda=5$. In terms of the mobility criterion for overconstrained mechanisms, the mobility of this $6 \mathrm{R}$ linkage is $m=5(6-$ $6-1)+6=1$.

\subsection{The coplanar constraint with axes lying in a plane}

Constraint singularity of the plano-spherical linkage occurs when it moves to the configuration in Fig. 2(b) with axes of all revolute joints coplanar and axes of $R_{4}$ and $R_{1}$ collinear. In the singular configuration, there are four joint axes intersecting at common point $A$ and four joint axes in parallel. Specifically, axes of joints $\mathrm{R}_{1}, \mathrm{R}_{2}, \mathrm{R}_{4}$ and $\mathrm{R}_{6}$ have common point $A$ and axes of joints $\mathrm{R}_{1}, \mathrm{R}_{3}, \mathrm{R}_{4}$ and $\mathrm{R}_{5}$ are parallel. In this singular configuration, the mechanism motion-screw system degenerates since all motion screws distributed on a single plane. The degeneration means the order of the mechanism screw-system changes to $d_{t}=\operatorname{dim}\left(\mathbb{S}_{t}\right)=3$. Consequently, the instantaneous mobility of the linkage in this singular position becomes $m=3(6-6-1)+6=3$. It implies the linkage obtains two more infinitesimal motions.

\subsection{The concurrent constraint with finite intersection of axes}

Considering the Grassmann varieties of lines having a finite intersection in 3D space and the disposition of joint axes on the single plane in the singular position in Fig. 2(b), the plano-spherical linkage is able to move out of the transitory position and keep $\mathrm{R}_{1}, \mathrm{R}_{2}, \mathrm{R}_{4}$ and $\mathrm{R}_{6}$ intersecting at point $A$ in Fig. 2(c). Under such a condition, joints $\mathrm{R}_{3}$ and $\mathrm{R}_{5}$ whose axes do not pass point $A$ are geometrically restricted and the degree-of-freedom of these two joints changes to zero. This means there are only four active revolute joints and four effective bodies (links). It further implies the parameter $n$ in mobility criterion changes to 4 . As a result, the mobility of the linkage in this evolved motion branch $\mathrm{MB}_{1}$ is calculated as $m=3(4-4-1)+4=1$. 


\subsection{The parallel constraint with intersections of axes at infinity}

The plano-spherical linkage is able to move out of the transitory position and keep $R_{1}, R_{3}, R_{4}$ and $R_{5}$ parallel to each other in Fig. $2(d)$, which corresponds to the Grassmann varieties of parallel lines in 3D space. Under such a condition, joints $R_{2}$ and $R_{6}$ are then geometrically restricted and their degree-of-freedom changes to zero simultaneously. This leads to motion branch $\mathrm{MB}_{2}$ with four effective bodies and four active revolute joints in parallel. The mobility of the linkage in this evolved motion branch $\mathrm{MB}_{2}$ is calculated as $m=3(4-4-1)+4=1$.

\subsection{The coincident constraint with collinear axes}

Since axes of joints $R_{4}$ and $R_{1}$ are collinear in the transitory position, the plano-spherical linkage is also able to change to motion branch $\mathrm{MB}_{3}$ which is a serial chain with all bodies rotating around those two aligned axes. In this motion branch, the evolved serial chain implements rotary motion as a compound revolute joint in Fig. 2(e). Joints $R_{2}, R_{3}, R_{5}$ and $R_{6}$ are geometrically restricted and the degrees-of-freedom of each joint changes to zero, leading to an evolved serial chain with mobility one.

Above analyses reveal that geometric constraints of joint axes corresponding to Grassmann varieties of lines allow the linkage to pass its singular position and to reconfigure to a spherical 4R linkage, a planar 4R linkage and a serial kinematic chain. Mobility of distinct motion branches varies within the scope of the infinitesimal mobility of the transitory position.

\section{Geometric Constraints Induced Reconfiguration of a Novel $7 \mathrm{R}$ Linkage}

This section presents a novel 7R linkage which is extracted from an asymmetric kirigami base in Fig. 3(a) and explores its reconfigurable motion branches. As illustrated in Fig. 3(b), links and revolute joints of the 7R linkage are denoted by $\mathrm{L}_{i}$ and $\mathrm{R}_{i}(i=1,2, \cdots, 7)$, respectively. In this $7 \mathrm{R}$ linkage, joint axes at distal ends of each link are coplanar, i.e. either having a common point or in parallel. Points $O, A, B, C$ and $D$ are the common points of successive axes in pairs. As the $7 \mathrm{R}$ linkage is a kinematic equivalent of the kirigami base in Fig. 3(a), common point $C$ coincides with $O$ when the linkage is in the flattened configuration with axes of all revolute joints located in a single plane. The twisting angle, $\alpha_{i}$, between each pair of 
adjacent axes are specified by $\alpha_{2}=\alpha_{4}=\alpha_{6}=\alpha_{7}=45^{\circ}, \alpha_{1}=\alpha_{5}=90^{\circ}$ and $\alpha_{3}=0^{\circ}$ since axes of joints $\mathrm{R}_{3}$ and $\mathrm{R}_{4}$ are parallel.
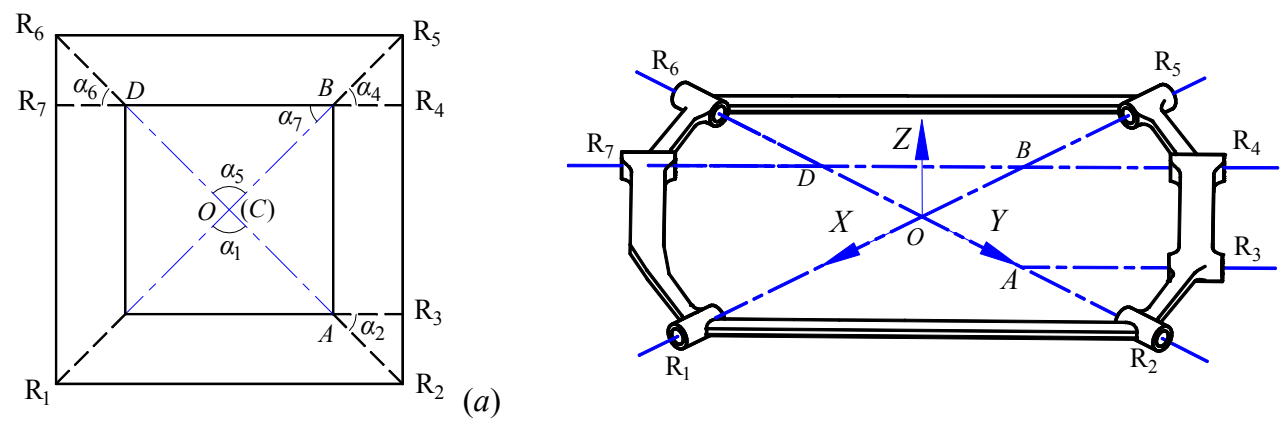

(b)

Figure 3: A kirigami base and its equivalent mechanism: (a) a kirigami base with seven creases, (b) the equivalent 7R linkage in the flattened configuration

When the closed-loop 7R linkage is in a general configuration away from the flattened configuration in Fig. 3(b), the order of mechanism motion-screw system is 6 and the mobility of the linkage is calculated as $m=6(7-7-$ 1) $+7=1$.

\subsection{Coplanar Constraints Induced Singular Configuration and the Evolved Serial Chain with Mobility One}

In the flattened configuration in Fig. 3, three pairs of the coplanar axes are collinear in particular, meaning $R_{1}$ and $R_{5}, R_{2}$ and $R_{6}$, as well as $R_{4}$ and $R_{7}$ are coaxial simultaneously. The mechanism motion-screw system of this configuration is a three-system due to the geometric condition with coplanar axes. Considering this peculiar disposition of all seven joint axes, the $7 \mathrm{R}$ linkage is able to move out of the singular configuration in three different ways.

When the linkage moves out of the singular configuration in Fig. 3 by rotating around collinear axes of joint $R_{1}$ and $R_{5}$, it reconfigures to motion branch $\mathrm{MB}_{1}$ with all other joints restricted, meaning the serial kinematic chain in Fig. 4(a).

Alternatively, once the linkage moves out of the singular configuration by rotating around collinear axes of joint $R_{2}$ and $R_{6}$, it reconfigures to motion branch $\mathrm{MB}_{2}$, the serial kinematic chain in Fig. 4(b). 

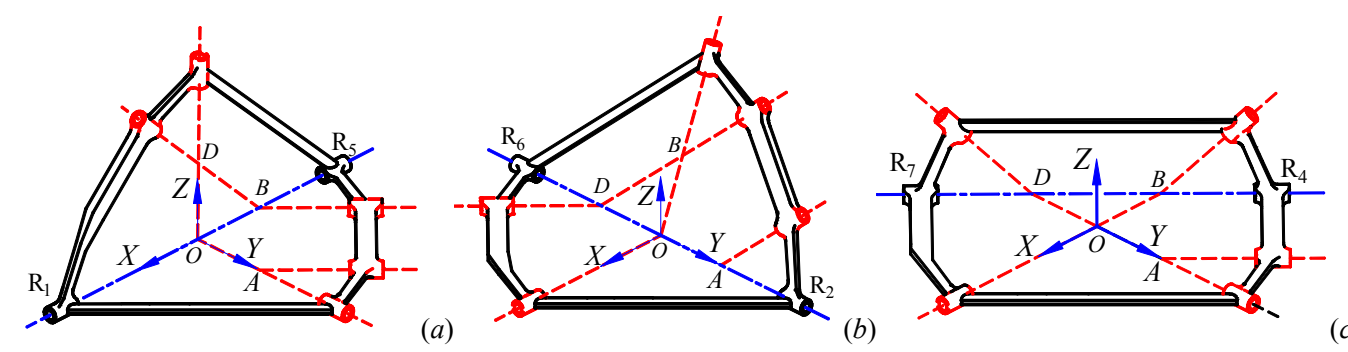

Figure 4: The evolved serial kinematics in three different motion branches: (a) $\mathrm{MB}_{1}$, (b) $\mathrm{MB}_{2}$, (c) $\mathrm{MB}_{3}$

As axes of joints $R_{4}$ and $R_{7}$ are coaxial in the singular configuration in Fig. 3, the linkage can also move out of this peculiar configuration by rotating around these two collinear axes, resulting to motion branch $\mathrm{MB}_{3}$ which is the serial kinematic chain in Fig. 4(c).

\subsection{Coplanar Constraints Induced Singular Configuration and the Evolved Overconstrained 6R Linkage}

When the serial kinematic chain in Fig. 4(c) moves to the flattened configuration in Fig. 5, axes of all revolute joints are coplanar again. In particular, axes of joints $R_{1}$ and $R_{6}$ are in parallel as well as that of $R_{2}$ and $R_{5}$. In this configuration, axes of joint $\mathrm{R}_{1}$ and $\mathrm{R}_{7}$ pass common point $B$ while axes of joints $\mathrm{R}_{2}$ and $R_{4}$ intersect at common point $D$ simultaneously. Apart from the axis of joint $R_{3}$, the disposition of other joint axes is with bilateral symmetry with respect to the plane passing point $O$ and perpendicular to line $B D$.

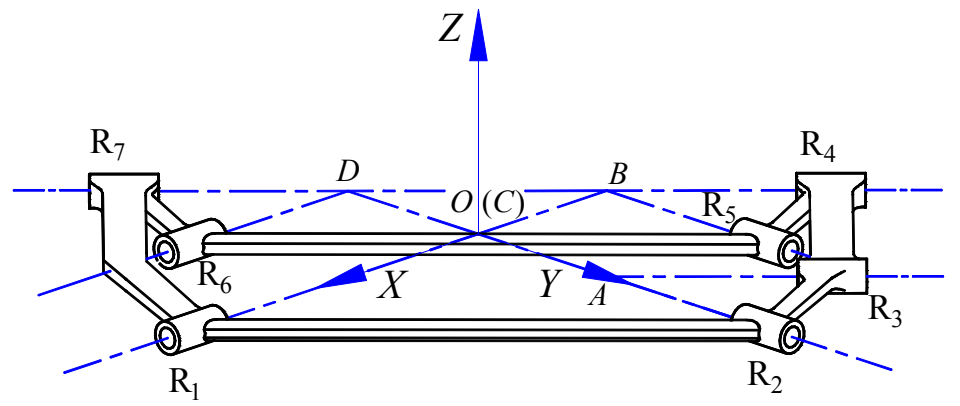

Figure 5: The flattened configuration of the $7 \mathrm{R}$ linkage with axes of joints $\mathrm{R}_{4}$ and $R_{7}$ collinear 
Since all joint axes are coplanar, motion screws of the $7 \mathrm{R}$ linkage at the configuration in Fig. 5 span a three-system, meaning $d_{t 1}=3$.

Considering the disposition of joint axes in Fig. 5, the 7R linkage is able to move to motion branch $\mathrm{MB}_{4}$ in Fig. 6 , where axes of joints $\mathrm{R}_{1}, \mathrm{R}_{7}$ and $\mathrm{R}_{6}$ remain coplanar and points $O$ and $C$ are disjointed. Resultantly, joint $\mathrm{R}_{7}$ is geometrically restricted with its degree-of-freedom change to zero, leading to six effective revolute joints. In this motion branch, axes of joints $R_{1}, R_{2}$ and $R_{3}$ are parallel to axes of $R_{6}, R_{5}$ and $R_{4}$, respectively.

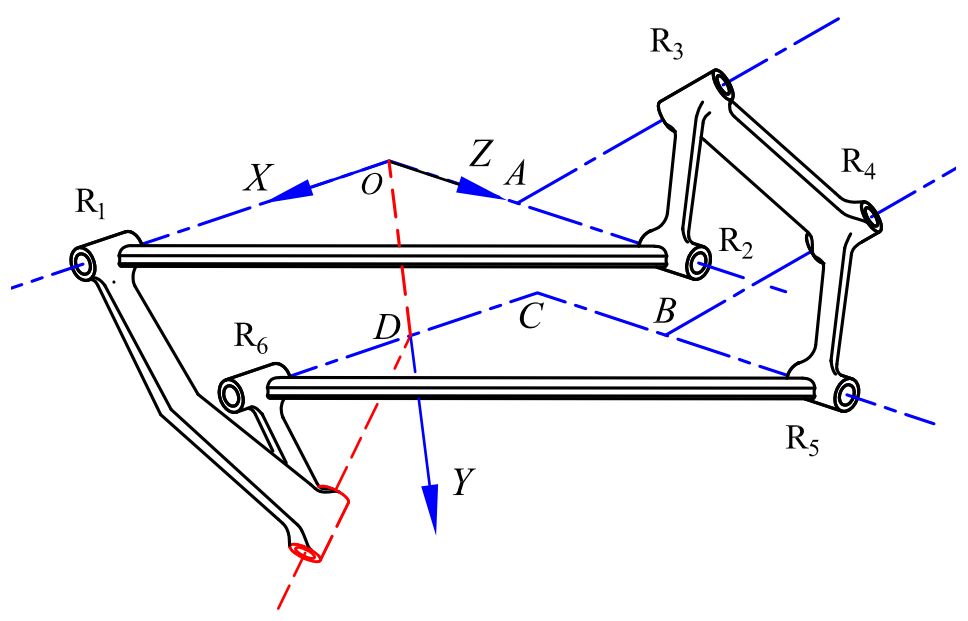

Figure 6: Motion branch $\mathrm{MB}_{4}$ : a overconstrained 6R linkage evolved from the $7 \mathrm{R}$ linkage

A coordinate frame $O-X Y Z$ is set up by aligning $X$-axis to axis of joint $\mathrm{R}_{1}$ and $Y$-axis to line $O D$ in Fig. 6 . The $Z$-axis is perpendicular to the plane formed by axes of joints $\mathrm{R}_{1}$ and $\mathrm{R}_{6}$. Accordingly, the mechanism motionscrew system expressed in frame $O-X Y Z$ is

$$
\mathbb{S}_{1}=\left\{\begin{array}{l}
\mathbf{S}_{1}=\left[\begin{array}{llllll}
1 & 0 & 0 & 0 & 0 & 0
\end{array}\right]^{\mathrm{T}} \\
\mathbf{S}_{2}=\left[\begin{array}{llllll}
0 & r c \theta_{1} & r s \theta_{1} & 0 & 0 & 0
\end{array}\right]^{\mathrm{T}} \\
\mathbf{S}_{3}=\left[\begin{array}{llllll}
l_{3} & l_{3} & n_{3} & r p & r l_{3} s \theta_{1} & -r l_{3} c \theta_{1}
\end{array}\right]^{\mathrm{T}} \\
\mathbf{S}_{4}=\left[\begin{array}{llllll}
l_{3} & l_{3} & n_{3} & r\left(n_{3}+p\right) & r\left(n_{3}+l_{3} s \theta_{1}\right) & -r l_{3}\left(2+c \theta_{1}\right)
\end{array}\right]^{\mathrm{T}} \\
\mathbf{S}_{5}=\left[\begin{array}{llllll}
0 & r c \theta_{1} & r s \theta_{1} & r^{2} s \theta_{1} & r^{2} s \theta_{1} & -r^{2} c \theta_{1}
\end{array}\right]^{\mathrm{T}} \\
\mathbf{S}_{6}=\left[\begin{array}{llllll}
1 & 0 & 0 & 0 & 0 & -r
\end{array}\right]^{\mathrm{T}}
\end{array}\right.
$$


where $p=\left(n_{3} c \theta_{1}-l_{3} s \theta_{1}\right), r$ is the distance between points $A$ and $O, \theta_{1}$ is the joint variable of $R_{1}$ and $\left[l_{3}, l_{3}, n_{3}\right]^{\mathrm{T}}$ is the unit vector pointing in the direction of screw $\mathbf{S}_{3}$. Given points $O$ and $C$ are coincident in Fig. 3, position vector of point $C$ is $[-r, r, 0]^{\mathrm{T}}$.

The mechanism constraint-screw system of the evolved $6 \mathrm{R}$ linkage is reciprocal to motion-screw system $\mathbb{S}_{1}$ and derived as

$$
\mathbb{S}_{1}^{\mathrm{c}}=\mathbf{S}_{1}^{\mathrm{r}}=\left[\begin{array}{lllll}
-1 & 1 & 0 & 0 & \frac{r s \theta_{1}\left(p-l_{3} s \theta_{1}\right)}{l_{3} s \theta_{1}-n_{3} c \theta_{1}} \\
r c \theta_{1}\left(1+\frac{l_{3}}{l_{3}-n_{3} c \theta_{1}}\right.
\end{array}\right]^{\mathrm{T}}
$$

The above constraint implies that motion-screw system of the linkage in Fig. 6 changes to $d_{1}=\operatorname{dim}\left(\mathbb{S}_{1}\right)=6-\operatorname{dim}\left(\mathbb{S}_{1}^{c}\right)=6-1=5$. There are only six joints of the original $7 \mathrm{R}$ linkage active in this motion branch and the finite mobility of the evolved $6 \mathrm{R}$ linkage is $m=5(6-6-1)+6=1$.
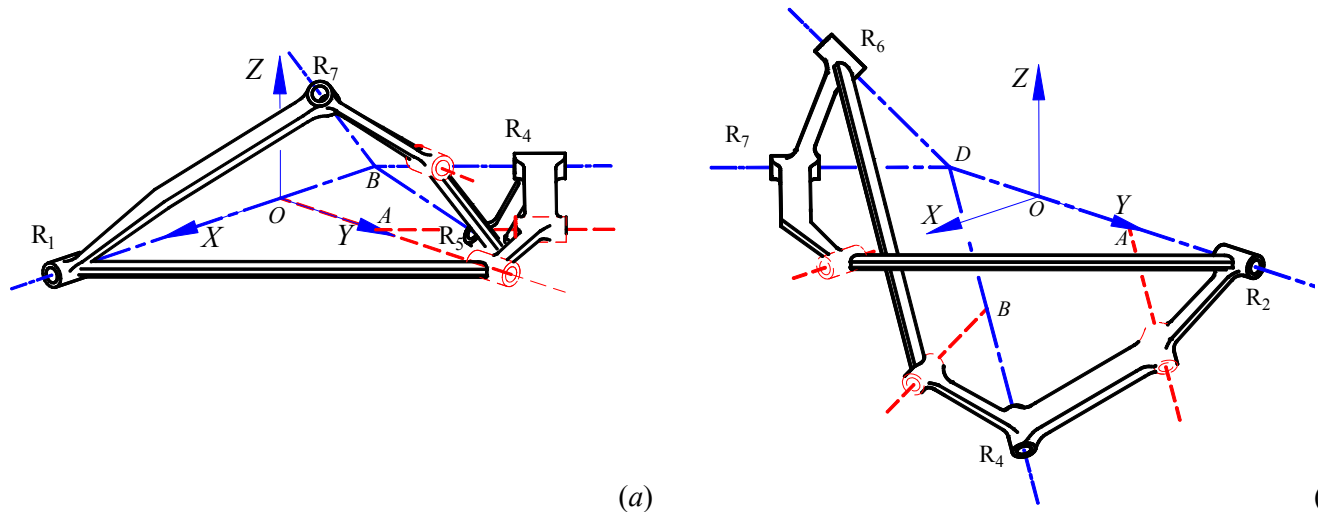

(a)

Figure 7: Two evolved spherical 4R linkages: (a) motion branch $\mathrm{MB}_{5}$ with spherical centre $B,(\mathrm{~b})$ motion branch $\mathrm{MB}_{6}$ with spherical centre $D$

\subsection{Concurrent Constraints Induced Spherical $4 R$ Linkages}

In the flattened singular configuration in Fig. 5 , axes of joints $R_{1}, R_{4}$, $\mathrm{R}_{5}$ and $\mathrm{R}_{7}$ have common point $B$. The $7 \mathrm{R}$ linkage is able to move to motion branch $\mathrm{MB}_{5}$ in Fig. 7(a) by passing this flattened transitory position. In this evolved motion branch, axes of joints $R_{1}, R_{2}, R_{3}$ and $R_{4}$ are coplanar while axes of joints $R_{5}, R_{6}$ and $R_{7}$ become coplanar simultaneously. In such a case, only joints $R_{1}, R_{4}, R_{5}$ and $R_{7}$ are active and the motion of remaining joints are geometrically restricted, allowing the linkage to work as a spherical $4 \mathrm{R}$ linkage with spherical centre $B$. 
The $7 \mathrm{R}$ linkage is also able to move to motion branch $\mathrm{MB}_{6}$ in Fig. 7(b) by passing this flattened transitory while keeping all links rotating about the common point $D$. In this evolved motion branch, axes of all seven joints are distributed in four planes intersecting at common point $D$. In this configuration, only joints $R_{2}, R_{4}, R_{6}$ and $R_{7}$ are active and the motion of other joints are geometrically restricted, allowing the linkage to perform as a spherical 4R linkage with spherical centre $D$.

When the 7R linkage moves to the configuration in Fig. 8, all seven joint axes are coplanar with axes of joints $R_{3}$ and $R_{7}$ aligned to each other. Axes of joints $R_{1}$ and $R_{6}$ as well as that of joints $R_{2}$ and $R_{5}$ are in parallel, respectively. Further, the former two parallel axes are perpendicular to the later two. These two sets of axes are symmetric with respect to two collinear axes of joints $R_{3}$ and $R_{7}$ which are parallel to axis of joint $R_{4}$.

As all seven axes are coplanar, the motion screws in this flattened configuration span a three-system. It implies that the motion-screw system of the $7 \mathrm{R}$ linkage degenerates from a five-system to a three-system, meaning $d_{t 2}=d_{t}-\tau_{2}=6-3=3$.

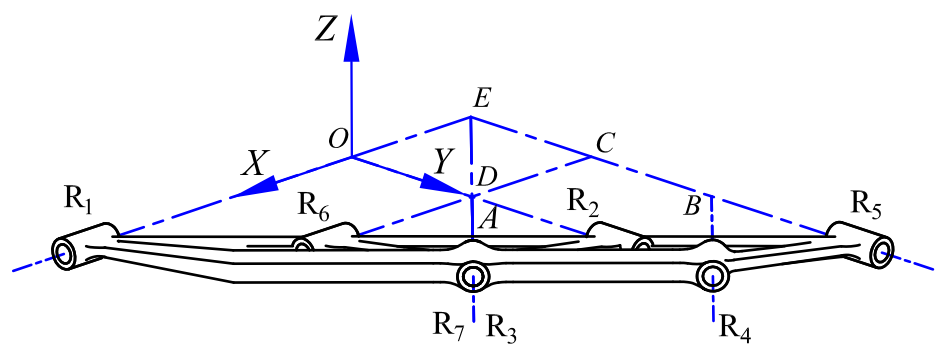

Figure 8: The transitory position of the $7 \mathrm{R}$ linkage with axes of joints $R_{3}$ and $\mathrm{R}_{7}$ collinear

As illustrated in Fig. 8, axes of joints $R_{1}, R_{3}, R_{5}$ and $R_{7}$ intersect at point $E$ in the flattened transitory position. By passing the transitory position, the $7 \mathrm{R}$ linkage is able to move to motion branch $\mathrm{MB}_{7}$ in Fig. 9(a). In this motion branch, axes of joints $R_{1}, R_{2}$ and $R_{3}$ remain coplanar. In the meantime, axes of $R_{3}, R_{4}$ and $R_{5}$ and axes of joints $R_{5}, R_{6}$ and $R_{7}$ located in another two planes. Hence, only joints $R_{1}, R_{3}, R_{5}$ and $R_{7}$ are active and remaining joints are geometrically restricted, allowing the linkage to perform as a spherical 4R linkage with spherical centre $E$.

By passing the flattened transitory position in Fig. 8 and keeping all links rotating about common point $A$, the $7 \mathrm{R}$ linkage is able to move to 


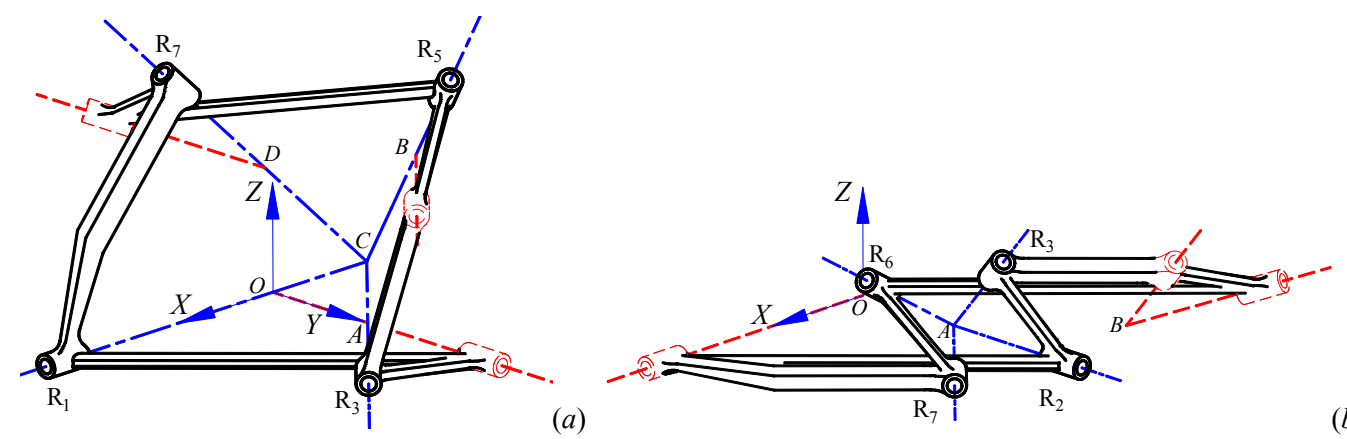

Figure 9: Two evolved spherical 4R linkages: (a) motion branch $\mathrm{MB}_{7}$ with spherical centre $E$, (b) motion branch $\mathrm{MB}_{8}$ with spherical centre $A$

motion branch $\mathrm{MB}_{8}$ in Fig. 9(b), where axes of joints $R_{3}, \mathrm{R}_{4}, \mathrm{R}_{5}$ and $\mathrm{R}_{6}$ and those of joints $R_{5}, R_{6}$ and $R_{7}$ are confined in two planes. Consequently, only joints $R_{2}, R_{3}, R_{6}$ and $R_{7}$ are active and the motion of remaining joints are geometrically restricted, allowing the linkage to work as a spherical $4 \mathrm{R}$ linkage with spherical centre $A$.

For all the evolved motion branches working as spherical $4 \mathrm{R}$ linkages with distinct spherical centres, there are four effective bodies and four active revolute joints in each motion branch. Hence, mobility of these evolved motion branches are calculated as $m=3(4-4-1)+4=1$.

\section{A Series of Reconfiguration of the Line- and Plane- Symmetric Bricard 6R Linkage}

\subsection{Geometry of the Line- and Plane-Symmetric Bricard 6R Linkage}

According to the kinematic structure of the special Bricard 6R linkage in Fig. 10, axes of joints $\mathrm{R}_{2}$ and $\mathrm{R}_{3}$ have common point $A$ and those of joints $\mathrm{R}_{5}$ and $\mathrm{R}_{6}$ have common point $B$. In a general configuration, axes of all six joints have three more common points, which are point $C$ of joints $\mathrm{R}_{2}$ and $\mathrm{R}_{6}$, point $D$ of joints $\mathrm{R}_{3}$ and $\mathrm{R}_{5}$ and point $E$ of joints $\mathrm{R}_{1}$ and $\mathrm{R}_{4}$. Point $O$ is the mid-point of line segment $A B$. Further, points $C$ and $D$ are located in plane $\Pi_{2}$, determined by intersecting axes of joint $\mathrm{R}_{1}$ and $\mathrm{R}_{4}$. Points $A$, $B, E$ and $O$ are coplanar and located in plane $\Pi_{1}$, which is perpendicular to plane $\Pi_{2}$. In this configuration, disposition of all six axes are with both rotational symmetry about axis $E O$ and bilateral symmetry with respect to two orthogonal planes $\Pi_{1}$ and $\Pi_{2}$. A Cartesian coordinate frame $O-X Y Z$ with 
the origin attached at mid-point $O$ is set as global frame of the $6 \mathrm{R}$ linkage. The $X$-axis is aligned with line $A B, Y$-axis is parallel to line $C D$ and $Z$-axis is collinear with common line $O E$ of two planes $\Pi_{1}$ and $\Pi_{2}$ following the right-hand rule.

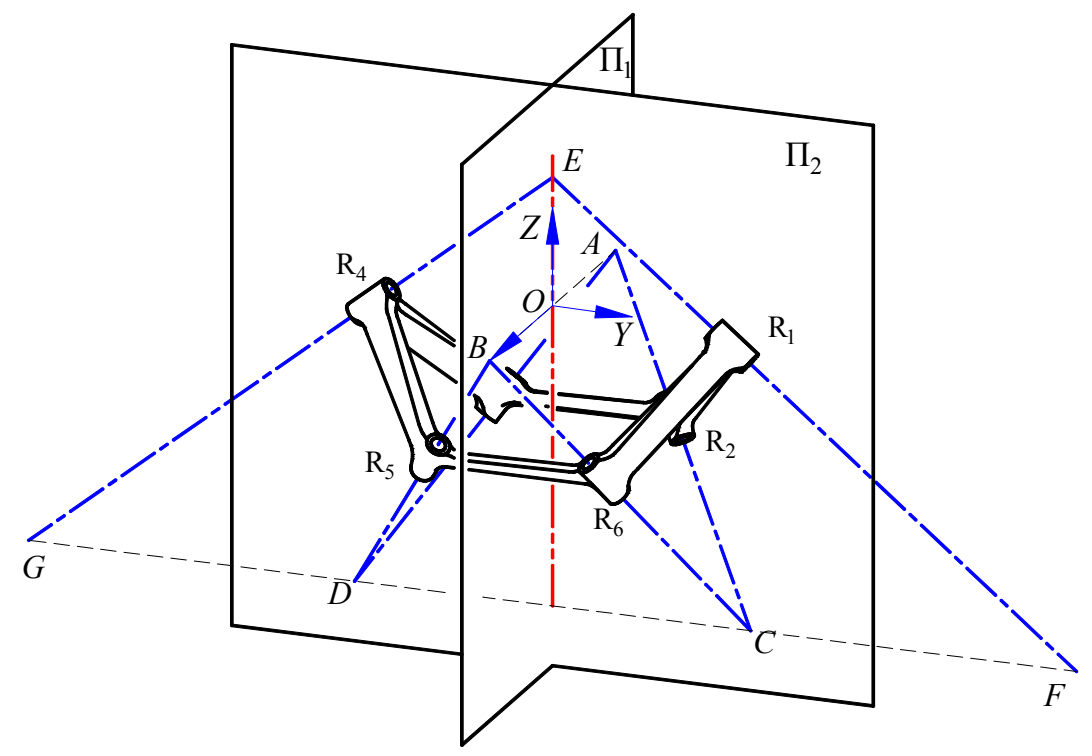

Figure 10: A line- and plane-symmetric Bricard 6R linkage

Considering the symmetric structure of the $6 \mathrm{R}$ linkage, position vectors of points $A, B, C, D, E, F$ and $G$ expressed in global frame $O-X Y Z$ are

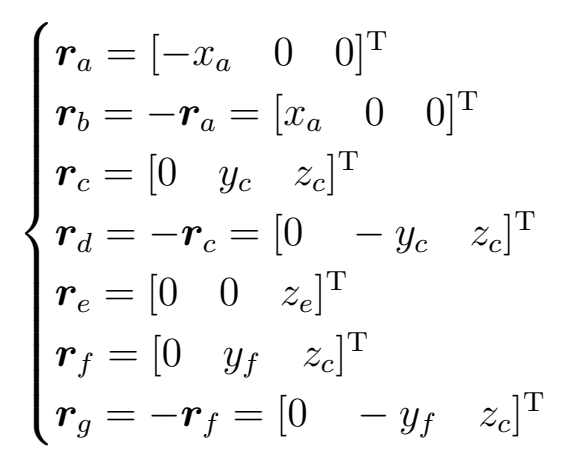

With position vectors in Eq. (8), motion screws of all six revolute joints 
expressed in global frame $O-X Y Z$ can be yielded as

$$
\mathbb{S}=\left\{\begin{array}{l}
\mathbf{S}_{1}=\left[\begin{array}{lllllll}
0 & -y_{f} & z_{e}-z_{c} & z_{e} y_{f} & 0 & 0
\end{array}\right]^{\mathrm{T}} \\
\mathbf{S}_{2}=\left[\begin{array}{lllllll}
x_{a} & -y_{c} & -z_{c} & 0 & x_{a} z_{c} & -x_{a} y_{c}
\end{array}\right]^{\mathrm{T}} \\
\mathbf{S}_{3}=\left[\begin{array}{lllllll}
x_{a} & y_{c} & -z_{c} & 0 & x_{a} z_{c} & x_{a} y_{c}
\end{array}\right]^{\mathrm{T}} \\
\mathbf{S}_{4}=\left[\begin{array}{lllllll}
0 & y_{f} & z_{e}-z_{c} & -y_{f} z_{e} & 0 & 0
\end{array}\right]^{\mathrm{T}} \\
\mathbf{S}_{5}=\left[\begin{array}{lllllll}
-x_{a} & y_{c} & -z_{c} & 0 & -x_{a} z_{c} & -x_{a} y_{c}
\end{array}\right]^{\mathrm{T}} \\
\mathbf{S}_{6}=\left[\begin{array}{lllllll}
-x_{a} & -y_{c} & -z_{c} & 0 & -x_{a} z_{c} & x_{a} y_{c}
\end{array}\right]^{\mathrm{T}}
\end{array}\right.
$$

The motion screws in Eq. (9) span a five-system. The constraint screw is reciprocal to motion screws and are derived as

$$
\mathbb{S}^{\mathrm{c}}=\mathbf{S}_{1}^{\mathrm{r}}=\left[\begin{array}{llllll}
0 & 1 & 0 & -z_{c} & 0 & 0
\end{array}\right]^{\mathrm{T}}
$$

The constraint screw in Eq. (10) represents a constraint force collinear with $C D$. This constraint force can also be obtained geometrically since a zero-pitch screw has to be coplanar with its reciprocal screws. The constraint screw that satisfies this geometric condition in the context of reciprocal screws has to be intersecting with all six motion screws, i.e., the screw whose axis passes points $F, C, D$ and $G$.

The motion-screw system and constraint-screw system in Eqs. (9) and (10) indicate that $d=\operatorname{dim}(\mathbb{S})=5$ and $\lambda=\operatorname{dim}\left(\mathbb{S}^{c}\right)=1$ and the $6 \mathrm{R}$ linkage is a overconstrained closed-loop mechanism. Following the mobility criterion for overconstraind mechanism, the mobility of the 6R linkage in Fig. 10 is calculated as $m=5(6-6-1)+6=1$.

\subsection{Motion Branch Changes in Concurrent Constraints Induced Spherical $4 R$ Linkages}

The line- and plane-symmetric 6R linkage in Fig. 10 moves to transitory configuration in Fig. 11 when common point $A$ coincides with point $B$. In this transitory configuration, axes of joints $R_{2}, R_{3}, R_{5}$ and $R_{6}$ intersect at point $O$. Accordingly, motion screws in Eq. (9) changes to

$$
\mathbb{S}_{t 2}=\left\{\begin{array}{l}
\mathbf{S}_{t 1}=\left[\begin{array}{lllllll}
0 & s \beta & c \beta & -z_{e} \beta & 0 & 0
\end{array}\right]^{\mathrm{T}} \\
\mathbf{S}_{t 2}=\left[\begin{array}{llllll}
-c \gamma & s \gamma & z & 0 & 0 & 0
\end{array}\right]^{\mathrm{T}} \\
\mathbf{S}_{t 3}=\left[\begin{array}{lllllll}
-c \gamma & -s \gamma & z & 0 & 0 & 0
\end{array}\right]^{\mathrm{T}} \\
\mathbf{S}_{t 4}=\left[\begin{array}{lllllll}
0 & -s \beta & c \beta & z_{e} s \beta & 0 & 0
\end{array}\right]^{\mathrm{T}} \\
\mathbf{S}_{t 5}=\left[\begin{array}{lllllll}
c \gamma & -s \gamma & z & 0 & 0 & 0
\end{array}\right]^{\mathrm{T}} \\
\mathbf{S}_{t 6}=\left[\begin{array}{llllll}
c \gamma & s \gamma & z & 0 & 0 & 0
\end{array}\right]^{\mathrm{T}}
\end{array}\right.
$$


where $\gamma$ is the angle measured between $X$-axis and the plane determined by axes of joints $R_{6}$ and $R_{3}$, while $\beta$ is the angle measured between screw axis $\mathrm{R}_{1}$ and $Z$-axis.
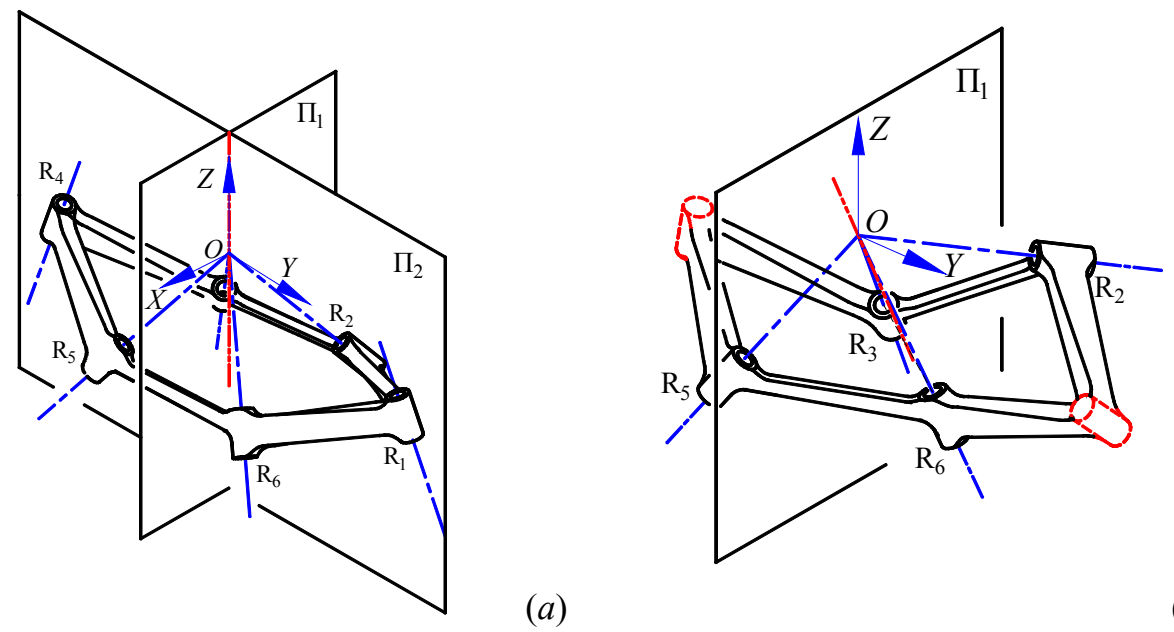

(a)

(b)

Figure 11: Reconfiguration from the $6 \mathrm{R}$ linkage to motion branch $\mathrm{MB}_{1}$ : (a) transitory position with axes of joints $R_{1}$ and $R_{4}$ having a common point on positive $Z$ axis, (b) the evolved spherical $4 \mathrm{R}$ linkage

Constraint screws of the linkage in its singular configuration in Fig. 11 (a) are reciprocal to motion screws in Eq. (11) and is derived as

$$
\mathbb{S}_{t 2}^{\mathrm{c}}=\left\{\begin{array}{l}
\mathbf{S}_{t 1}^{\mathrm{r}}=\left[\begin{array}{llllll}
0 & 1 & 0 & 0 & 0 & 0
\end{array}\right]^{\mathrm{T}} \\
\mathbf{S}_{t 2}^{\mathrm{r}}=\left[\begin{array}{llllll}
0 & 0 & 1 & 0 & 0 & 0
\end{array}\right]^{\mathrm{T}}
\end{array}\right.
$$

These two constraint screws in Eq. (12) represent two constraint forces collinear with $Y$ - and $Z$-axis. This implies that only four of motion screws in Eq. (11) are independent and the screw system in Eq. (9) degenerates. Thus, $d_{t 1}=$ $\operatorname{dim}\left(\mathbb{S}_{t 2}\right)=4$ and $\lambda=\operatorname{dim}\left(\mathbb{S}_{t 2}^{c}\right)=2$.

Since axes of joints $\mathrm{R}_{2}, \mathrm{R}_{3}, \mathrm{R}_{5}$ and $\mathrm{R}_{6}$ have common point $O$, the linkage is able to change to motion branch $\mathrm{MB}_{1}$ in Fig. 11 (b) by passing the configuration in Fig. 11(a) and keeping four axes intersecting at common point $O$.

In this motion branch, joints $\mathrm{R}_{1}$ and $\mathrm{R}_{4}$ are geometrically restricted and their degrees-of-freedom change to zero. The geometric condition of bilateral symmetry with respect to planes $\Pi_{1}$ and $\Pi_{2}$ breaks but the spherical $4 \mathrm{R}$ 

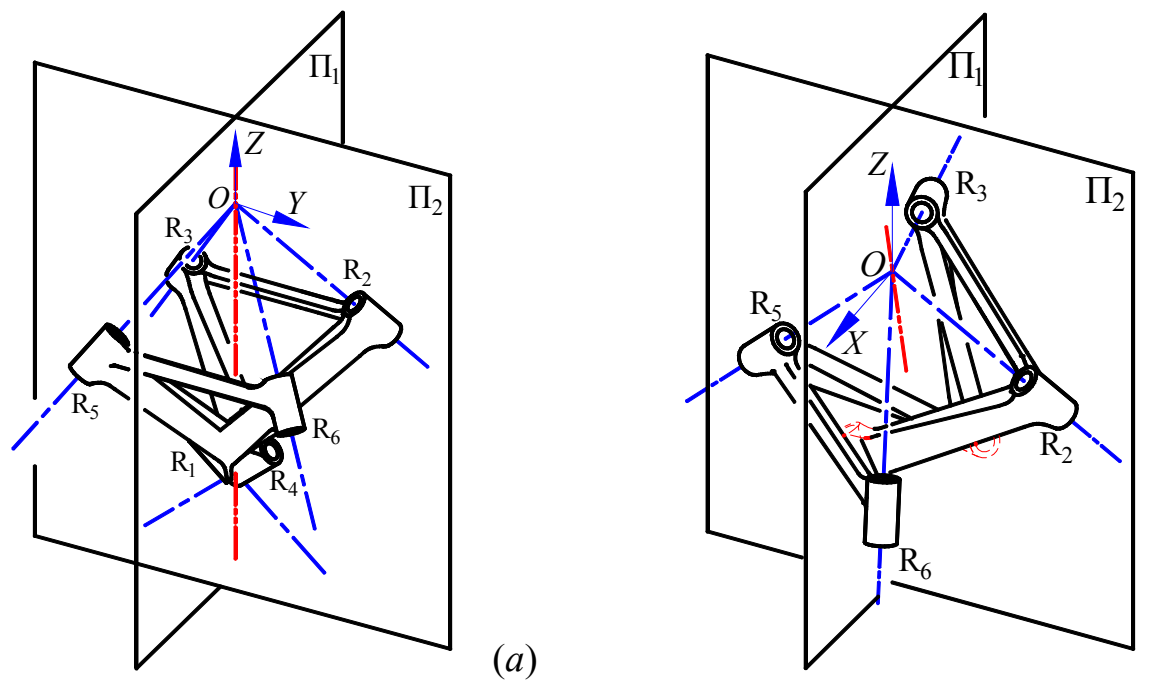

(b)

Figure 12: Reconfiguration from the $6 \mathrm{R}$ linkage to motion branch $\mathrm{MB}_{2}$ : (a) transitory position with axes of joints $R_{1}$ and $R_{4}$ having a common point on the negative $Z$ axis, (b) the evolved spherical $4 \mathrm{R}$ linkage

linkage is with rotational symmetry about the common line of the planes determined by $R_{2}$ and $R_{5}$ and that by $R_{3}$ and $R_{6}$. It derives that $d_{1}=$ $\operatorname{dim}\left(\mathbb{S}_{2}\right)=3$ and $\lambda_{2}=\operatorname{dim}\left(\mathbb{S}_{2}^{c}\right)=3$. There are only four active links $(n=4)$ and four active joints $(g=4)$ in this motion branch. The mobility of the evolved $4 \mathrm{R}$ linkage in this motion branch is calculated as $m=3(4-4-1)+4=$ 1.

In analogous to the transitory configuration above, the $6 \mathrm{R}$ linkage is able to move from a general line and plane symmetric configuration to transitory configuration in Fig. 12(a). In this specific configuration, axes of joints $\mathrm{R}_{2}$, $\mathrm{R}_{3}, \mathrm{R}_{5}$ and $\mathrm{R}_{6}$ meet at point $O$ while axes of joint $\mathrm{R}_{1}$ and $\mathrm{R}_{4}$ meet on the negative $Z$-axis. The constraint screws of the transitory configuration in Fig. 12(a) are same as that given in Eq. (12), meaning two constraint forces collinear with $Y$ - and $Z$-axis. It further implies that the motion-screw system of this configuration also degenerates, leading to $d_{2}=\operatorname{dim}\left(\mathbb{S}_{t 3}\right)=4$ and $\lambda_{3}=\operatorname{dim}\left(\mathbb{S}_{t 3}^{\mathrm{c}}\right)=2$.

When the linkage moves out of this transitory configuration without breaking the concurrent geometric constraint of having common point $O$, it changes to motion branch $\mathrm{MB}_{2}$ in Fig. 12(b) where the linkage works as a spherical $4 \mathrm{R}$ linkage with joints $\mathrm{R}_{1}$ and $\mathrm{R}_{4}$ in Fig. 12(a) geometrically 
restricted.
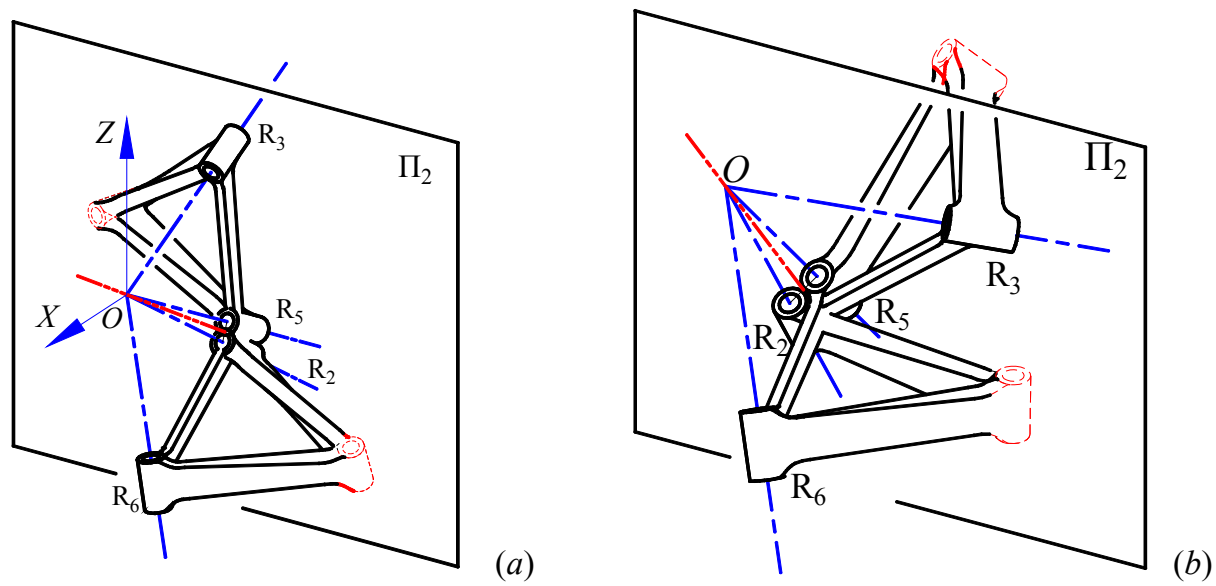

Figure 13: Reconfiguration from the $6 \mathrm{R}$ linkage to motion branch $\mathrm{MB}_{3}$ : (a) transitory position with four coplanar axes of joints $R_{2}, R_{3}, R_{5}$ and $R_{6}$ coplanar, (b) the evolved spherical $4 \mathrm{R}$ linkage with bilateral symmetry

In particular, the spherical $4 \mathrm{R}$ linkages in motion branches $\mathrm{MB}_{1}$ and $\mathrm{MB}_{2}$ are able to move to transitory configuration in Fig. 13(a) where axes of active joints $R_{2}, R_{3}, R_{5}$ and $R_{6}$ are coplanar. In this transitory configuration, the motion screw-system further degenerates and changes to a two-system, i.e. $d_{t 3}=\operatorname{dim}\left(\mathbb{S}_{t 4}\right)=2$ and $\lambda=\operatorname{dim}\left(\mathbb{S}_{t 4}^{c}\right)=4$. By passing this flattened configuration, the linkage is able to change to motion branch $\mathrm{MB}_{3}$ in a planesymmetric configuration in Fig. 13(b). In other words, axes of joints $R_{5}$ and $\mathrm{R}_{6}$ are mirror of $\mathrm{R}_{2}$ and $\mathrm{R}_{3}$ with respect to a plane passing $O P$. In this planesymmetric configuration, $d_{4}=\operatorname{dim}\left(\mathbb{S}_{4}\right)=3$ and the mobility of the linkage is one.

\section{Conclusions}

This paper explored the essence of reconfigurable single-loop kinematic chains with mobility one. In terms of changes of four variables $d, n, g$ and $f_{i}$ in the Chebychev-Grübler-Kutzbach mobility criterion, motion branch variations of reconfigurable mechanisms were classified into four general categories. In particular, reconfigurations corresponding to variation of parameter $d$ were revealed with consideration of degeneration of degree-of-freedom of certain revolute joints in single-loop kinematic chains with mobility one. 
With the essence of reconfiguration, the interrelationship of motion-branch changes through constraint singularity induced transitory positions was investigated in the context of reciprocity of screws. Based on screw theory, four types of geometric constraints leading to transitory positions were explored by analysing motion branch changes of the Bennett plano-spherical linkage in conjunction with Grassmann varieties. Geometric constraints induced screw-system variation and consequent motion branch changes were interpreted with a novel asymmetric $7 \mathrm{R}$ linkage and the line- and planesymmetric Bricard 6R linkage. These analyses of the $7 \mathrm{R}$ linkage and the Bricard linkage further validated the classification and the interrelationship of screw-system variation in motion branch changes of reconfigurable single-loop linkages. The present work paves a way of analysing reconfigurable singleloop kinematic chains based on constraint singularity induced screw-system variation. The essence of reconfiguration and the geometric constraints based approach can be further extended to synthesis of reconfigurable single-loop mechanisms.

\section{Acknowledgement}

The authors thank the European Commission for the support in the human-robot interaction project SQUIRREL in the name of Clearing Clutter Bit by Bit under Grant No. 610532 and the support of the National Natural Science Foundation of China under Grant No. 51205016.

\section{References}

[1] K. Wohlhart, Kinematotropic linkages, Recent Advances in Robot Kinematics (1996) 359-368.

[2] G. Gogu, Branching singularities in kinematotropic parallel mechanisms, Proceedings of the 5th International Workshop on Computational Kinematics (2009) 341-348.

[3] C. M. Gosselin, J. Angeles, Singularity analysis of closed-loop kinematic chains, IEEE Trans. Rob. Auto. 6 (3) (1990) 281-290.

[4] J. S. Dai, Conceptual study of the dexterous reconfigurable assembly and packaging system, Recent Advances in Robot Kinematics (PS960326). 
[5] J. S. Dai, J. R. Jones, Mobility in metamorphic mechanisms of foldable/erectable kinds, ASME J. Mech. Des. 121 (3) (1999) 375-382.

[6] L. P. Zhang, J. S. Dai, Reconfiguration of spatial metamorphic mechanisms, ASME J. Mech. and Rob. 1 (1) (2009) 011012.

[7] C. H. Liu, T. L. Yang, Essence and characteristics of metamorphic mechanisms and their metamorphic ways, Proc.11th World Congress in Mechanism and Machine Science (2004) 1285-1288.

[8] D. Zlatanov, I. A. Bonev, C. M. Gosselin, Constraint singularities as c-space singularities, Advances in Robot Kinematics (2002) 183-192.

[9] X. Kong, C. M. Gosselin, P. L. Richard, Type synthesis of parallel mechanisms with multiple operation modes, ASME J. Mech. Des. 129 (7) (2007) 595-601.

[10] H.-S. Yan, C.-H. Kuo, Topological representations and characteristics of variable kinematic joints, ASME J. Mech. Des. 128 (2) (2006) 384-391.

[11] H.-S. Yan, C.-H. Kang, Configuration synthesis of mechanisms with variable topologies, Mech. Mach. Theory 44 (5) (2009) 896-911.

[12] C. G. P. Fanghella, E. Giannotti, Parallel robots that change their group of motion, Advances in Robot Kinematics (2006) 49-56.

[13] K. Zhang, J. S. Dai, Y. F. Fang, A new metamorphic mechanism with ability for platform orientation switch and mobility change, ASME/IFToMM International Conference on Reconfigurable Mechanisms and Robots (2009) 626-632.

[14] D. M. Gan, J. S. Dai, Q. Z. Liao, Mobility change in two types of metamorphic parallel mechanisms, ASME J. Mech. Rob. 1 (4) (2009) 041007.

[15] K. Zhang, J. S. Dai, Y. F. Fang, Topology and constraint analysis of phase change in the metamorphic chain and its evolved mechanism, ASME J. Mech. Des. 132 (12) (2010) 121001.

[16] D. Tosi, G. Legnani, N. Pedrocchi, P. Righettini, H. Giberti, Cheope: A new reconfigurable redundant manipulator, Mech. Mach. Theory 45 (4) (2010) 611-626. 
[17] Q. Zeng, Y. F. Fang, K. F. Ehmann, Design of a novel 4-dof kinematotropic hybrid parallel manipulator, ASME J. Mech. Des. 133 (12) (2011) 121006.

[18] L. Carbonari, M. Callegari, G. Palmieri, M.-C. Palpacelli, A new class of reconfigurable parallel kinematic machines, Mech. Mach. Theory 79 (2014) 173-183.

[19] A. Moosavian, F. Xi, Design and analysis of reconfigurable parallel robots with enhanced stiffness, Mech. Mach. Theory 77 (2014) 92-110.

[20] C. Galletti, P. Fanghella, Single-loop kinematotropic mechanisms, Mech. Mach. Theory 36 (2001) 743-761.

[21] K. Zhang, J. S. Dai, A kirigami-inspired 8r linkage and its evolved overconstrained $6 \mathrm{r}$ linkages with the rotational symmetry of order two, ASME J. Mech. Rob. 6 (2) (2014) 021007.

[22] C. Kuo, H. Yan, On the mobility and configuration singularity of mechanisms with variable topologies, ASME. J. Mech. Des. 129 (2006) 617624 .

[23] X. Kong, C. Huang, Type synthesis of single-dof single-loop mechanisms with two operation modes, ASME/IFToMM International Conference on Reconfigurable Mechanisms and Robots (2009) 136-141.

[24] X. Kong, M. Pfurner, Type synthesis and reconfiguration analysis of a class of variable-dof single-loop mechanisms, Mech. Mach. Theory 85 (2015) 116-128.

[25] X. He, X. Kong, D. Chablat, S. Caro, G. Hao, Kinematic analysis of a single-loop reconfigurable $7 \mathrm{r}$ mechanism with multiple operation modes, Robotica 32 (2014) 1171-1188.

[26] K. Zhang, A. Müller, J. S. Dai, A novel reconfigurable 7r linkage with multifurcation, Recent Advances in Robot Kinematics I (2015) 15-25.

[27] Y. Chen, Z. You, Two-fold symmetrical 6r foldable frames and their bifurcations, International Journal of Solids and Structures 46 (2009) $4504-4514$. 
[28] Y. Chen, W. Chai, Bifurcation of a special line and plane symmetric bricard linkage, Mech. Mach. Theory 46 (2011) 515-533.

[29] J. E. Baker, An analysis of bricard linkages, Mech. Mach. Theory 15 (1980) 267-286.

[30] K. Zhang, J. S. Dai, Trifurcation of the evolved sarrus-motion linkage based on parametric constraints, Advances in Robot Kinematics (2014) $345-353$.

[31] K. Hunt, Kinematic Geometry of Mechanisms, Oxford University Press, London, 1978.

[32] P. Lopez-Custodio, J. Rico, J. Cervantes-Sanchez, G. Perez-Soto, Reconfigurable mechanisms from the intersection of surfaces, ASME J. Mech. Rob. 8 (2) (2016) 021029.

[33] R. S. Ball, A Treatise on the Theory of Screws, Cambridge University Press, Massachusetts, 1900.

[34] J. McCarthy, Introduction to Theoretical Kinematics, MIT Press, Cambridge, 1990.

[35] J. S. Dai, Geometric Foundation and Screw Algebra for Mechanisms and Robotics, Higher Education Press, Beijing, 2014.

[36] A. Kecskemthy, M. Hiller, Automatic closed-form kinematics-solutions for recursive single-loop chains, Proceedings of the 22nd Biennial ASMEMechanisms Conference on Flexible Mechanisms, Dynamics, and Analysis 47 (1992) 387-393.

[37] A. Kecskemthy, On closed form solutions of multiple-loop mechanisms, Computational kinematics (1993) 263-274.

[38] C. G. Gibson, K. H. Hunt, Geometry of screw systems-2. classification of screw systems, Mech. Mach. Theory 25 (1) (1990) 11-27.

[39] J. M. R. Martinez, J. Duffy, Classification of screw systemsi. one-and two-systems, Mech. Mach. Theory 27 (4) (1992) 459-470.

[40] J. M. R. Martinez, J. Duffy, Classification of screw systemsii. threesystems, Mech. Mach. Theory 27 (4) (1992) 471-490. 
[41] J. S. Dai, J. R. Jones, Interrelationship between screw systems and corresponding reciprocal systems and applications, Mech. Mach. Theory 36 (5) (2001) 633-651.

[42] J. S. Dai, J. R. Jones, Null-space construction using cofactors from a screw algebra context, Proceedings of the Royal Society of London, Series A: Mathematical, Physical and Engineering Sciences 458 (2024) (2002) 1845-1866.

[43] J. S. Dai, Z. Huang, H. Lipkin, Mobility of overconstrained parallel mechanisms, ASME. J. Mech. Des. 128 (1) (2006) 220-229.

[44] J. P. Merlet, Singularity configurations of parallel manipulators and grassmann geometry, Int. J. Rob. Res. 8 (9) (1989) 45-56.

[45] T. Mruthyunjaya, Kinematic structure of mechanisms revisited, Mech. Mach. Theory 38 (4) (2003) 279-320.

[46] G. Gogu, Mobility of mechanisms: a critical review, Mech. Mach. Theory 40 (9) (2005) 1068-1097.

[47] K. Waldron, The constraint analysis of mechanisms, J. Mech. 1 (1966) 101-114.

[48] K. H. Hunt, Screw axes and mobility in spatial mechanisms via the linear complex, J. Mech. 2 (1967) 307-327.

[49] Y. Qin, J. S. Dai, G. Gogu, Multi-furcation in a derivative queer-square mechanism, Mech. Mach. Theory 81 (2014) 36-58.

[50] G. T. Bennett, The parallel motion of sarrus and some allied mechanisms, Philosophy Magazine, 6th series 9 (1905) 803-810. 